\title{
Naringenin, a Flavonoid, Modulates Gut Microbiome and Ameliorates Hormone Levels to Improve Polycystic Ovary Syndrome in Letrozole-induced Rats
}

\section{Yan-Xiang Wu}

BUCM: Beijing University of Chinese Medicine

Xiu-Yan Yang

BUCM: Beijing University of Chinese Medicine

Yuan-yuan Hu

BUCM: Beijing University of Chinese Medicine

Tian An

BUCM: Beijing University of Chinese Medicine

Bo-Han LV

BUCM: Beijing University of Chinese Medicine

Juan Lian

BUCM: Beijing University of Chinese Medicine

Ting-Ye Wang

BUCM: Beijing University of Chinese Medicine

Xue-Li Bao

BUCM: Beijing University of Chinese Medicine

Jia-Jian Zhu

BUCM: Beijing University of Chinese Medicine

Lin Gao

BUCM: Beijing University of Chinese Medicine

Guangjian Jiang ( $\sim$ bucmjiang@163.com )

School of Traditional Chinese Medicine, Beijing University of Chinese Medicine, Beijing, China

\section{Research}

Keywords: Polycystic ovary syndrome, naringenin, gut microbiome, sex hormone, glucose metabolic, beneficial microbes

Posted Date: October 19th, 2020 
DOI: https://doi.org/10.21203/rs.3.rs-92829/v1

License: (c) (1) This work is licensed under a Creative Commons Attribution 4.0 International License. Read Full License 


\section{Abstract}

Background: Polycystic ovary syndrome (PCOS) is a common female endocrinopathy, which severely affect the menstruation and fertility of patients. Naringenin, a natural flavanone, has emerged as a potential therapeutic agent for the management a variety of diseases. However, the underlying mechanism of naringenin in anti-PCOS is unclear. This study was focused on investigating the effects of naringenin on body weight, ovarian tissue, serum hormone level, glucose metabolism level and gut microbiome in letrozole-induced PCOS model rats.

Methods: First, we administered letrozole gavage to 10-week-old SD female rats for 4 weeks to induce PCOS rats model, the estrus cycle was observed through the vaginal smear of rats to determine the establishment successful of a PCOS rat model. Then, the successfully modeled PCOS rats were treated with naringenin for 2 months. Finally, observed the changes of rat body weight, ovarian tissue, serum hormone level, glucose metabolism level and gut microbiome after naringenin treatment.

Results: The naringenin treatment ameliorate the hormone levels, such testosterone (T), estradiol (E2), follicle-stimulating hormone ( $\mathrm{FSH})$, luteinizing hormone (LH), improve insulin resistance and the ovarian tissue pathological changes, reduced body weight in the PCOS model rat. Meanwhile, through the detection of rDNA in the faeces of the PCOS model rat, we found some beneficial microbes such [Ruminococcus], Faecalibacterium, Butyricimonas, Lachnospira, Parabacteroides, Butyricicoccus and Roseburia were enrichment in naringenin group when compared with the PCOS rats.

Conclusions: In summary, our results indicated that naringenin could play an anti-PCOS role, and its mechanism may be closely related to regulating the beneficial microbes of gut microbiome. Our research could provide a new perspective for the treatment of PCOS and its related disease.

\section{Background}

Polycystic ovary syndrome (PCOS) is one of the most common endocrine diseases in women of childbearing age, with the prevalence of 6-21\% [1]. Its main features are anovulation, hyperandrogenism, polycystic ovarian morphology, and the main clinical manifestations include menstrual disorders, infertility, hirsutism, metabolic syndrome comprising obesity, dyslipidemia, insulin resistance (IR) [2], which places women at an increased risk for development into cardiovascular disease and diabetes mellitus [3]. Although several studies have confirmed that genetic, gestational environment and lifestyle factors are connected with the development of PCOS [4-6], the exact underlying mechanism triggers for these key biochemical and metabolic disorders in PCOS is still largely unclear. Moreover, due to the side effects of anti-androgens or ovarian function inhibitors, the clinical use of such drugs is generally not accepted. Because development the effective drugs or operations are still a challenge in the treatment of PCOS, other therapies need to be used. So, it is necessary to formulate alternative treatment strategies for women with PCOS, more and more studies using natural plant-based products to treat PCOS. 
In recent years, the gut microbiome has become a key factor affecting health and disease, and has made major contributions to metabolic diseases. Studies showed that the gut microbiota can induce or regulate insulin resistance and chronic inflammation, and can also regulate sex hormone levels [7-9]. Meanwhile, with the deepening of research, accumulating evidence showed that intestinal flora is closely related to the occurrence and development of PCOS $[1,8,10]$. Such as, transplanting feces of PCOS patients into mice can cause PCOS phenotype in mice [10], both PCOS patients and animal models, PCOS-induced changes in the gut microbiota alpha and beta diversity can occur to a certain extent [3]. In addition, the clinical manifestations of PCOS were significantly reduced in obese women treated with low-fat diet [11], and emerging evidence showed that the composition of the intestinal flora may affect women's metabolic homeostasis and reproductive fitness [12]. Based on the above research, we calculate that the gut microbiome may be play an important role to PCOS.

Naringenin, a citrus flavonoid that possesses various biological activities, which can be extracted from grapefruit and widely exists in the plant kingdom, and has been isolated from several plants [13, 14], and the natural plant extract of naringenin has emerged as a potential therapeutic agent for the management a variety of diseases[15]. Meanwhile, it has been shown that naringenin regulates lipoprotein metabolism, it may be used in the management of diabetes, atherosclerosis and insulin resistance, which was extensively discussed in a previous review [16]. Similarly, naringenin has been shown to have hypoglycemic activity in type 2 diabetic rats [17]. In addition, naringenin has good prospects as an ideal therapeutic agent, a study showed that naringenin treatment in a rat model of PCOS significantly increased the levels of the reactive oxygen species (ROS) scavenging enzymes catalase (CAT), superoxide dismutase (SOD), glutathione peroxidase (GPX), prevented weight increase and reduced serum glucose levels, normalized estradiol and testosterone levels and maintained the normal anatomy of the ovaries $[18,19]$. However, whether and how naringenin regulates the diversity of gut microbiome to anti-PCOS is still unknown. Therefore, we aimed to study the role of naringenin in regulating gut microbiome, glucose metabolism, sex hormone levels, ovarian morphology and body weight of letrozoleinduced PCOS rat, to initially reveal the potential mechanism of its anti-PCOS effect.

\section{Materials And Methods}

\subsection{Animal}

Female Sprague-Dawley (SD) rats, ten-week-old (body weight, 270-300 g), were specific pathogen-free (SPF) level inbred and obtained from the professional Laboratory Animal Company (Beijing Vital River Laboratory Animal Technology Co., Ltd.). They were randomly divided into two groups, including normal group $(n=6)$ and PCOS group $(n=24)$. All rats need 2 weeks to adapt the new environment before the experiment was started, all animals were fed in standard animal room (room temperature: $22 \pm 2 \circ \mathrm{C}$; relative humidity: $30-40 \%)$, free dry pellet diet and water were supply. All protocols in this study were approved by the Animal Care Committee of Beijing University of Chinese Medicine (BUCM), China, and these animals were treated according to the Animal Care Committee. 


\subsection{Induction of PCOS and Diane-35 and Naringenin drugs administration}

All animals were under a periodic cycle, the letrozole (Jiangsu Hengrui Medicine co.,Itd, gyzz $\mathrm{H} 199991001$ ), with the concentration of $1 \mathrm{mg} / \mathrm{kg}$, were gavage for rats to induce PCOS per day, with 28 consecutive days. The estrus cycle was monitored by using vaginal smear, and observation changes in the estrous cycle under the microscope. After, the 18 successful letrozole-induced PCOS rats were randomly divided into the PCOS group (MX), Diane-35 group (YY), naringenin group (YP) with 6 rats in each group. The Diane-35(Bayer Weimar GmbH und Co.KG, gyzz j20140114) as a positive control group $(0.2 \mathrm{mg} / \mathrm{kg} / \mathrm{day})$ and the naringenin (BioRuler, USA,100 Mill Plain Road Danbury, CT06811) as a treatment group ( $20 \mathrm{mg} / \mathrm{kg} /$ day) were respectively intragastric administration for YY and YP group. Meanwhile, the normal group (ZC) and PCOS group (PCOS) were given same volume of saline. During the whole experiment period, the body weight (BW) was checked every week, the fasting blood glucose (FBG) was tested after the animals were ambrosia for eight hours. With the experiment termination, all animals were sacrificed in anesthetized deeply, the serum, bilateral ovaries, kidney, and liver were collected for further detection.

\subsection{Hematoxylin and Eosin(HE) staining}

With $4 \%$ paraformaldehyde were used to fixed ovaries tissue and then embedded in paraffin, sliced for 5 microns, staining with hematoxylin and eosin (H\&E). Finally, these tissue samples were removed under a light microscope (Olympus, Japan) with blindly observe and photograph. All histological experimentation were manipulated at the specialties laboratory of BUCM.

\subsection{Serum hormone analysis}

The blood samples of trunk circulation were successfully collected and allowed to stand at room temperature for 2 hours for incubation. All samples were centrifuged ( $3,000 \mathrm{rpm}, 15 \mathrm{~min}, 4 \circ \mathrm{C})$, then the serum was collected into $1.5 \mathrm{ml}$ EP tube, kept at $-80 \circ \mathrm{C}$ for subsequent experiments.

The basic serum hormone testing including testosterone ( $T$ ), estradiol (E2), follicle-stimulating hormone $(\mathrm{FSH})$, luteinizing hormone (LH), insulin resistance index(FINS). All serum levels were measured by using the radio Immunosorbent Assay (Tianjin Jiuding Medical Bioengineering Co., Ltd.). According to the specifications to use all kits, repeatability analysis for each sample. The homeostasis model of assessment for insulin resistance (HOMA-IR) and insulin sensitivity index (ISI) are an effective way to determine the insulin-resistance and insulin sensitivity index in the rat model. So, the HOMA-IR was calculated according to (FINS $[\mu \mathrm{IU} / \mathrm{ml}] \times \mathrm{FBG}[\mathrm{mmol} / \mathrm{L}] / 22.5)$, and the ISI was calculated according to 1/[FBG $(\mathrm{mmol} / \mathrm{l}) \times \mathrm{FINS}(\mu \mathrm{lU} / \mathrm{ml})][20]$.

\subsection{Fecal DNAextraction and 16SrDNA high-throughput sequencing}

\subsubsection{Sample collection}


We collected fresh fecal samples from four groups rats before all animals were sacrificed, fresh fecal were placed in a $1.5 \mathrm{ml}$ sterile EP tube with quickly freeze in liquid nitrogen, then store at $-80{ }^{\circ} \mathrm{C}$ waiting for further experiments.

\subsubsection{Fecal DNA extraction and PCR amplification}

Total microbial genomic DNA samples were extracted using the DNeasy PowerSoil Kit (QIAGEN, Inc., Netherlands), following the manufacturer's instructions, stored at $-20^{\circ} \mathrm{C}$ before further analysis. The quantity and quality of extracted DNAs were measured using a NanoDrop ND-1000 spectrophotometer (Thermo Fisher Scientific, Waltham, MA, USA) and 1.2\% agarose gel electrophoresis, respectively. Microbial DNA was extracted from fecal samples using the Illumina company TruSeq Nano DNA LT Library Prep Kit (Qiagen, Valencia, CA, United States), according to manufacturer's protocols. The V3-V4 region of the bacteria 16S ribosomal RNA genes was amplified by PCR ( $98 \circ \mathrm{C}$ for 2 min, followed by 25 cycles at $98 \circ \mathrm{C}$ for $15 \mathrm{~s}, 55 \circ \mathrm{C}$ for $30 \mathrm{~s}$, and $72 \circ \mathrm{C}$ for $30 \mathrm{~s}$, with a final extension at $72 \circ \mathrm{C}$ for $5 \mathrm{~min}$ ) using the forward primer 338F(5'-ACTCCTACGGGAGGCAGCA-3') and the reverse primer 806R(5'GGACTACHVGGGTWTCTAAT-3' ). The PCR components contained $5 \mu$ l of Q5 reaction buffer (5x), $5 \mu \mathrm{l}$ of Q5 High-Fidelity GC buffer (5x), $0.25 \mu$ l of Q5 High-Fidelity DNA Polymerase ( $5 \mathrm{U} / \mu \mathrm{l}), 2 \mu \mathrm{l}(2.5 \mathrm{mM})$ of dNTPs, $1 \mu \mathrm{l}(10 \mathrm{uM})$ of each Forward and Reverse primer, $2 \mu \mathrm{l}$ of DNA Template, and 8.75 $\mu$ of H 20.

\subsubsection{Sequencing and data process}

Sequence data analyses were mainly performed using QIIME and R packages (v3.2.0). In this study, the main selected sequencing analysis includes usable raw reads, operational taxonomic units (OTUs), species annotation (genus level), taxonomic_tree, alpha diversity analysis, OTU abundance Venn diagram and heat map of species composition. Species annotation: the top 20 species of taxonomic level of each group were plotted with accumulative histograms to visualize the species of relative abundance and their proportion at different taxonomic levels. The taxonomic_tree can display the composition of all taxa at the same time. Alpha analysis was analysis the species diversity of a single sample. In this study, Chao1 and Observed species indexes were used to indicate abundance, Shannon and Simpson indexes were used to indicate diversity, Faith's PD indexes characterizes the diversity based on evolution, Pielou's evenness indexes characterizes uniformity, Good's coverage indexes represents coverage. The different species analysis including OTU abundance Venn diagram and the heat map of species composition, the Venn diagram was to study which species were shared among different sample groups and which were unique, the heat map further compares the differences between species composition and samples, realized the display of the species abundance distribution trend of each sample.

\subsection{Statistical analysis}

The experimental data was processed by SPSS (version20.0, IBM SPSS Statistics), the results were expressed as mean \pm standard error of the man (SEM). Statistical differences were determined by Student's independent t-test or One-way ANOVA, the significance was accepted at $P$ values $<0.05$. Significance was shown as * $p<0.05$, ** $p<0.01$ vs normal group; \#p $<0.05$, \#\#p $<0.01$ vs PCOS group. 


\section{Results}

\subsection{Characteristic of Letrozole-induced PCOS rat model}

In this study, we used letrozole for 4 weeks to induce the PCOS rat model and monitored the estrous cycle through vaginal smears. The results showed that we successfully established a rat model of PCOS induced by letrozole, which was confirmed by the irregular estrous cycle (Fig. 1.A). Generally speaking, the estrous cycle was longer than 5 days, or there was no obvious cycle, or it continued to be in the per-estrus, or the interests' period, these are usually considered to be disordered. As shown in Fig. 1.A, the vaginal smear of the normal group showed an obvious and regular estrous cycle. But compared with the normal group, the estrus cycle showed irregular changes and the most common cell type was leukocytes under microscope, suggesting vaginal smears of letrozole-induced model group rats exhibited a disorder of the estrus cycle (Fig. 1.B).

\subsection{Effects of Naringenin on body weight in PCOS rats}

In order to observe the influence of naringenin on the body weight of PCOS rats, we conducted a statistical analysis of body weight in this experiment, founding the body weights of the PCOS group was significantly increased when compared to the normal group, $\left({ }^{*} p<0.05\right.$, ${ }^{\star \star} p<0.01 \mathrm{vs}$ control; $\# p<0.05$, $\# \# p<0.01$ vs PCOS). Interestingly, the naringenin groups exhibited obviously decreased than Diane-35 group when compare with the PCOS group, but the difference was not statistically significant. This suggests that naringenin had a good effect for lose weight on letrozole- induced PCOS rats by eight-week intervention.

\subsection{Effects of Naringenin on histological changes in PCOS}

\section{rats}

With $4 \%$ paraformaldehyde-fixed and paraffin-embedded of rat ovaries, sectioned at $5-\mu \mathrm{m}$ slices for H\&Estained (Fig. 3A). In PCOS group, the ovaries showed pathological changes, no or very few corpus luteum structures, a large number of expanded cystic follicles, no radioactive crowns and oocytes were seen, the ovaries showed typical polycystic changes. But the naringenin group had large number of corpus luteum and had different levels of follicle at different developmental stages, the granulosa cell layer was multilayered with neatly arranged and normal in structure. Based on the above description, we found the pathological changes of the ovarian tissue have been greatly improved after 8 weeks of treatment by naringenin. Meanwhile, the ovaries resembled with the naringenin rats in all aspects after Diane35treatment.

\subsection{Effect of Naringenin on serum hormonal level in PCOS rats}


To study whether naringenin could ameliorate letrozole-induced hormonal levels in PCOS rats, we tested levels of T, E2, FSH and $\mathrm{LH}$. The result showed that the levels of serum T, LH and FSH were significantly increased and the level of serum E2 were significantly reduced in the PCOS group when compared with the normal group. After 8 weeks treatment, we found that the levels of serum T, LH and FSH were significantly reduced and the level of serum E2 was significantly increased. $\left({ }^{*} p<0.05,{ }^{*} \mathrm{p}<0.01 \mathrm{vs}\right.$ control; \#p<0.05, \#\#p<0.01vs PCOS; Fig. 4.). However, it was noticed that compared to the PCOS group, only the level of serum E2 difference was not statistically significant by Diane-35 treatment.

\subsection{Effect of Naringenin on glucose metabolism in PCOS rats}

To explore whether naringenin could improve letrozole-induced metabolic abnormalities in PCOS rats, we tested levels of FPG and fasting insulin (FINS) and further evaluated the effect of naringenin treatment on IR and insulin sensitivity in PCOS rats. The result showed that the FBG and FINS levels were significantly increased when compared with the normal group, but after the naringenin treatment, the serum levels of FBG and FINS were significantly decreased when compared to the PCOS group $\left({ }^{*} p<0.05\right.$, ${ }^{*} p<0.01$ vs control; \#p<0.05, \#\#p<0.01vs PCOS;Fig. 5.A/B). The FBG level had a decline in the Diane-35 group, but the difference was not statistically significant. In addition, compared with the normal group, the HOMA-IR was significantly increased and ISI was significantly reduced in letrozole-induced PCOS group. Interestingly, the levels of HOMA-IR and ISI were changed significantly by naringenin treatment when compared with the PCOS group, including significantly decreased in HOMA-IR and significantly increased in ISI. ( ${ }^{\star} p<0.05$, ${ }^{\star *} p<0.01$ vs control; $\# p<0.05$, \#\#p<0.01vs PCOS;Figure.5.C,D).

\subsection{Effects of Naringenin on gut microbiome in PCOS rats 3.6.1 The statistics of sequencing}

We fulfilled gene sequencing on the V3-V4 region of 16S rRNA to assess the impact of naringenin on the dysbiosis of gut microbiome in letrozole-induced PCOS rats. In total, 233,2605 usable raw reads (200,6653 valid sequences), 113,7408 high-quality sequence reads, and 8,2283 OTUs with a similarity of $97 \%$ were received from all 24 samples.

The number of reads obtained from Daine-35 group was the highest (100134 \pm 2657$)$, while in naringenin group was the lowest (89284 \pm 3412 ) (Fig. 6A). On the genus level, the control group had the most OUTs (783.2 \pm 42.65$)$, after the treatment of naringenin, the number of OTUs decreased (OTU $(601.8 \pm 26.35)$ in naringenin group and (637.2 \pm 34.27$)$ in Daine-35 group) (Fig. 6B).

\subsubsection{Overall structural changes of gut microbiome}

In our study, we analyzed the taxonomic compositions of bacterial communities in genus level among the normal, PCOS, naringenin and Diane-35 groups. The proportion of the microbial genus in the naringenin 
group and Diane-35 were very different from the PCOS model rats. In naringenin group, the microbial genus of Oscillospira, Blautia, Helicobacter, [Ruminococcus], Coprococcus, Parabacteroides, Faecalibacterium, Paraprevotella were higher when compared with PCOS group. In Diane-35 group, the microbial genus of Oscillospira, Coprococcus, Allobaculum, Desulfovibrio, Streptococcus and [Prevotella] were higher than PCOS group (Fig. 6C). Meanwhile, we also analyzed the species classification hierarchy tree with abundance information, the naringenin group showed that the abundance of Faecalibacterium prausnitzi, Helicobacter rodentium, Ruminococcus albus, Ruminococcus flavefaciens, Blautia product, [Ruminococcus] gnavus, Prevotella copri and Helicobacter rodentium were significantly increase(species level) these were consistent with the genus level results. In Diane-35 group, we found the abundance of Ruminococcus bromii (species level), Anaerostipes, Turicibacter, Allobaculum, Desulfovibrio (genus level), Peptostreptococcaceae and Coriobacteriaceae (family level) were remarkably increase(Fig. 6D).

\subsubsection{The alpha diversity analysis}

According alpha diversity analysis to observe the Chao1 index, Simpson index, Shannon index, Pielou_e index, Observed_species index, Faith_pd index and Goods_coverage index, we found that the alpha diversities were significantly changed all groups. When compared with the control group, the Chao1 index, Observed_species index and Faith_pd index were significantly reduced, but the Goods_coverage index was significantly increased in other three groups. (Fig. 6E, $p<0.05$ ). While no difference was found for the Simpson's diversity index, Shannon index and Pielou_e index.

\subsubsection{Key phylotypes of gut microbiome corresponding to Naringenin treatment}

There was a clear difference in species difference analysis and marker species screen. First of all, the Venn diagram showed some common and unique species among different groups, as shown in Fig. 1.A, the unique species OTUS in the control, PCOS, Diane-35 and naringenin groups were respectively $9449(25.04 \%), 6534(17.32 \%), 7089(18.79 \%), 7212(19.11 \%)$, it was no difficult found that the unique species OTUS was increase after treatment by naringenin when compared with the PCOS group (Fig. 6F). Secondly, we analyzed the heat map that represent the gut microbiome changes on the genus level of different groups, it showed that some beneficial species were significantly abundant by naringenin treatment 8 weeks, such as the genus of Parabacteroides, Lactobacillus, Faecalibacterium, Bacteroides, Roseburia, Butyricicoccus, Streptococcus were enrichment. In the Diane-35 group, the Lactobacillus, Anaerostipe,[Prevotella]םBifidobacterium, Streptococcus were abundance. Interestingly, these beneficial species were not emerged in PCOS group (Fig. 6G).

\section{Discussion}

PCOS is a metabolic syndrome, obesity, chronic inflammatory, dyslipidemia and IR are usually accompanied $[2,21]$. In recent ten years, relevant population-scale studies have illustrated the potential role of the intestinal microbiome in changing the health of the host, and the human gut microbiome is necessary for maintaining the human health and immune system [22]. Recently, several studies have 
revealed intestinal flora is closely linked to the occurrence of PCOS, the diversity and composition of the gut microbiota will undergo certain changes when the case of metabolic disorders [23-25]. Previous studies have found that gut microbiome can induce or regulate insulin resistance, obesity and chronic inflammation, and can also regulate sex hormone levels [26, 27]. In our study, similar to the results of these studies, we found the naringenin treatment changed the intestinal flora, body weight, ovarian tissue morphology, the levels of hormone, glucose metabolism on letrozole-induce PCOS rats.

Consistent with existing literature, in the current study, we observed the irregular estrous cycles, the ovary pathological change, levels of sex hormone were abnormal such as $\mathrm{LH}$, estradiol, testosterone and FSH in PCOS group of letrozole-induced when compared with the normal group [19, 28, 29]. All those features illustrated the model of PCOS were established. Interestingly, a flavanone, naringenin extracted from citrus fruits and exist in many traditional Chinese herbal medicines, has been displayed to have multiple effective effects on cells both in vivo and in vitro [30]. The result of naringenin treatment was the estradiol level and ISI increased, the T, LH, FSH, FBG, FINS and HOMA-IR levels decreased when compared to the levels in PCOS group. It was noticed that naringenin treatment not only had a significant amelioration in serum hormone levels, but also improved insulin resistance, prevented weight increase and maintained the normal anatomy of the ovaries in rat model of letrozole-induced PCOS. Those changes showed that naringenin maybe play an important role to adjustment the hormone levels in treatment the pathological change of ovulation disorder in PCOS.

There was a connection between the PCOS and gut microbiome, studies showed that the intestinal microbiota can regulate sex hormone level in the host, such as a decrease in alpha diversity, the change key phylotypes of gut microbiome corresponding, an increase of beneficial bacteria [36, 37], In order to research the role of the intestinal microbiota between naringenin with PCOS, we used 16S rDNA analysis technology to evaluate changes in the intestinal flora with naringenin in the PCOS rats of letrozole-induce. In our study, observing the disorder in the gut microbiota and revealing changes in the relative abundances of specific species in the gut microbiome in naringenin group. We found 7 differentially abundant bacterial taxa in the naringenin group, the genus of [Ruminococcus], Faecalibacterium, Butyricimonas, Lachnospira, Parabacteroides, Butyricicoccus and Roseburia were enrichment, these also explain why the unique species OTUS was increasing after treatment by naringenin when compared with the PCOS group. The result showed the naringenin treatment can increase the proportion of beneficial bacteria, and normalized the intestinal flora.

Interestingly, in the species level of gut microbiome, we noticed that the PCOS rats lack beneficial microorganisms such Faecalibacterium prausnitzii and the disease-related microbes Prevotella copri was appeared. But the Faecalibacterium prausnitzii of beneficial microbes was significantly abundance in naringenin group. Gut microbes such as Faecalibacterium prausnitzii is generally regard as "good bacteria" that has function for health-promoting [32]. As well as, in naringenin group, in the genus level of gut microbiome, we found some new beneficial bacteria were enrichment, such as the microbial genus of Faecalibacterium, Roseburia and Parabacteroides which can reduce the inflammation and obesity to promote intestinal microbiota homeostasis [33, 34]. Meanwhile, The SCFA-producing bacteria Roseburia, 
Lachnospira, Butyricicoccus, Lactobacillus and the dominant genus associated with SCFAs including Streptococcus [35-37] were abundant after naringenin treatment. In addition, the bacteria Lachnospira and Roseburia of butyrate-producing were also more abundant in the naringenin group, and the content of butyric acid, a beneficial substance in the intestine, can prevent and treat obesity-related diseases [38]. Several studies have demonstrated that various benefits of probiotics to the host have been shown in numerous human clinical trials, the role of these organisms is to improve the balance of the intestinal flora and increase the production of short-chain fatty acids(SCFAs), which can directly or indirectly play the role of anti-inflammatory, protect the intestinal barrier function, regulate human metabolism and immunity [39-41]. Especially, (SCFAs) play an important role in the treatment of obesity, insulin resistance, diabetes and other metabolic diseases [42].As well as study showed that SCFAs can improve intestinal health by promoting the increase of intestinal barrier function and reducing the translocation of bacterial endotoxins on the intestinal wall, this is one of the reasons it reduces inflammation and improves insulin resistance [43]. So, we conjecture the levels of FBG, FINS and HOMA-IR declined, the level of ISI increase may be regulate by gut microbiome, finally contributes to improve insulin resistance. From what has been discussed above, we speculate that the regulation of gut gut microbiome may be a potential mechanism for naringenin to prevented weight increase, reduction in serum glucose levels, improve insulin resistance, and promoting ameliorate the hormone levels in the PCOS model rat.

\section{Conclusions}

In conclusion, our research showed naringenin can prevent letrozole-induced PCOS-related symptoms such as obesity, insulin resistance, and sex hormone disorders, which may be related to the alteration of gut microbiome. Although an ant-PCOS activity of naringenin has been reported, no study to focus on exploring the relationship between naringenin and gut microbiome of PCOS through 16S rRNA sequencing analysis. Our research fills this gap, it suggest naringenin may be a therapeutic effect on PCOS-related metabolic disorders by adjusting the composition of gut microbiome. This provides a new train of thought for the pathogenesis and treatment of PCOS. But its specific mechanism still needs further experiments, which is also our future research direction.

\section{Abbreviations}

Polycystic Ovary Syndrome (PCOS); Insulin Resistance (IR); Reactive Oxygen Species (ROS); Catalase (CAT); Superoxide Dismutase (SOD); Glutathione Peroxidase (GPX); Sprague-Dawley (SD); Specific Pathogen-Free (SPF); Body weight (BW); Fasting Blood Glucose (FBG); Figure(Fig); Cystic Follicle(CF); Corpus Luteum(CL); Normal Follicle(F); Oocyte(O); Hematoxylin and Eosin (H\&E); Corpora Luteums (CL); Beijing University of Chinese Medicine(BUCM); Testosterone(T); Estradiol (E2); Follicle-Stimulating Hormone (FSH); Luteinizing Hormone (LH); Insulin Resistance Index(FINS); Homeostasis Model of Assessment for Insulin Resistance (HOMA-IR); Insulin Sensitivity Index (ISI); PCOS group (MX); Diane-35 group (YY); Naringenin group (YP); Normal group (ZC); Quantitative Insights Into Microbial Ecology (QIIME); Operational Taxonomic Units (OTUs); Short-Chain Fatty Acids (SCFAs). 


\section{Declarations}

\section{Author contributions}

Guangjian Jiang and Lin Gao designed the experiments; Yanxiang Wu, Xiuyan Yang performed the experiments and wrote the manuscript. Yuanyuan Hu, Tian An, Bohan Lv, Juan Lian, TingYe Wang, XueLi Bao, JiaJian Zhu, analyzed the data, All authors reviewed the manuscript.

\section{Acknowledgments}

Not applicable

\section{Conflicts of interest}

The authors declare no conflicts of interest concerning the authorship and/or publication of this paper.

\section{Funding}

This work was supported by Grants from the Basic research projects in Beijing-TianjinHebei(19JCZDJC65400(Z)); Independent project for graduate students of Beijing University of Chinese Medicine(2020-JYB-XSCXCY-119); Independent project for graduate students of Beijing University of Chinese Medicine(2020-JYB-XSCXCY-007).

\section{References}

[1] A.E. Joham, H.J. Teede, S. Ranasinha et al , Prevalence of infertility and use of fertility treatment in women with polycystic ovary syndrome: data from a large community-based cohort study, J. Womens Health 24 (4) (2002) 299-307 2015.

[2] D.A. Dumesic, S.E. Oberfield, E. Stener-Victorin, J.C. Marshall, J.S. Laven, R.S. Legro, Scientific statement on the diagnostic criteria, epidemiology, pathophysiology, and molecular genetics of polycystic ovary syndrome, Endocr. Rev. 36 (5) (2015) 487-525.

[3] Thackray Varykina G.(2019). Sex, Microbes, and Polycystic Ovary Syndrome. Trends Endocrinol. Metab., 30(1), 54-65. doi:10.1016/j.tem.2018.11.001.

[4]Vink, J. M., Sadrzadeh, S., Lambalk, C. B., and Boomsma, D. I. (2006). Heritability of polycystic ovary syndrome in a Dutch twin-family study. J. Clin. Endocrinol. Metab. 91, 2100-2104. doi: 10.1210/jc.20051494

[5] Abbott, D. H., Dumesic, D. A., Eisner, J. R., Colman, R. J., and Kemnitz, J. W.(1998). Insights into the development of polycystic ovary syndrome (PCOS) from studies of prenatally androgenized female rhesus monkeys. Trends Endocrinol. Metab. 9, 62-67. doi: 10.1016/S1043-2760(98)00019-8 
[6] Zhang, C., Yin, A., Li, H., Wang, R., Wu, G., Shen, J., et al. (2015). Dietary modulation of gut microbiota contributes to alleviation of both genetic and simpleobesityinchildren.EBioMedicine2,968984.doi:10.1016/j.ebiom.2015.07.007

[7] Insenser María., Murri Mora., Del Campo Rosa., Martínez-García M Ángeles., Fernández-Durán Elena., Escobar-Morreale Héctor F.(2018). Gut Microbiota and the Polycystic Ovary Syndrome: Influence of Sex, Sex Hormones, and Obesity. J. Clin. Endocrinol. Metab., 103(7), 2552-2562. doi:10.1210/jc.2017-02799

[8] Cai Wei., Xu Jixiong., Li Gang., Liu Tao., Guo Xiali., Wang Huajie., Luo Liping.(2020). Ethanol extract of propolis prevents high-fat diet-induced insulin resistance and obesity in association with modulation of gut microbiota in mice. Food Res. Int., 130(undefined), 108939. doi:10.1016/j.foodres.2019.108939

[9] Qi Xinyu., Yun Chuyu., Sun Lulu., Xia Jialin., Wu Qing., Wang Ying., Wang Lina., Zhang Yangming., Liang Xianyi., Wang Liying., Gonzalez Frank J., Patterson Andrew D., Liu Huiying., Mu Liangshan., Zhou Zehong., Zhao Yue., Li Rong., Liu Ping., Zhong Chao., Pang Yanli., Jiang Changtao., Qiao Jie.(2019). Gut microbiota-bile acid-interleukin-22 axis orchestrates polycystic ovary syndrome. Nat. Med., 25(8), 12251233. doi:10.1038/s41591-019-0509-0

[10] Zhang Jiachao., Sun Zhihong., Jiang Shuaiming., Bai Xiaoye., Ma Chenchen., Peng Qiannan., Chen Kaining., Chang Haibo., Fang Tuanyu., Zhang Heping.(2019). Bifidobacterium lactisProbiotic V9 Regulates the Secretion of Sex Hormones in Polycystic Ovary Syndrome Patients through the Gut-Brain Axis. mSystems, 4(2), undefined. doi:10.1128/mSystems.00017-19

[11] Kiddy, D. S., Hamilton-Fairley, D., Bush, A., Short, F., Anyaoku, V., Reed, M. J., et al. Improvement in endocrine and ovarian function during dietary treatment of obese women with polycystic ovary syndrome. Clin. Endocrinol ,1992;36: 105-111.

[12]Silva Mauro S B., Giacobini Paolo.(2019). Don't Trust Your Gut: When Gut Microbiota Disrupt Fertility. Cell Metab., 30(4), 616-618. doi:10.1016/j.cmet.2019.09.005

[13] Ho PC, Saville DJ, Wanwimolruk S: Improved high-performance liquid chromatographic method for the analysis of Naringin in grapefruit juice without extraction. Pharmacy Pharmacol Commun, 1998; 4(10): 473-76

[14] Frabasile S, Koishi AC, Kuczera D et al: The citrus flavanone naringenin impairs dengue virus replication in human cells. Sci Rep, 2017; 7: 41864

[15]Zeng Wenfeng., Jin Lingtao., Zhang Fayun., Zhang Chunling., Liang Wei.(2018). Naringenin as a potential immunomodulator in therapeutics. Pharmacol. Res., 135(undefined), 122-126. doi:10.1016/j.phrs.2018.08.002

[16] E.E. Mulvihill, A.C. Burke, M.W. Huff, Citrus flavonoids as regulators of lipoprotein metabolism and atherosclerosis, Annu. Rev. Nutr. 36 (2016) 275-299. 
[17] McGrath JC, Drummond GB, McLachlan EM et al: Guidelines for reporting experiments involving animals: The ARRIVE guidelines. Br J Pharmacol, 2010; 160(7): 1573-76

[18] Lee MH, Yoon S, Moon JO. The flavonoid naringenin inhibits dimethylnitrosamine-induced liver damage in rats. Biol Pharm Bull 2004;27:72-6.

[19] Hong Yanli., Yin Yanyun., Tan Yong., Hong Ke., Zhou Huifang.(2019). The Flavanone, Naringenin, Modifies Antioxidant and Steroidogenic Enzyme Activity in a Rat Model of Letrozole-Induced Polycystic Ovary Syndrome. Med. Sci. Monit., 25(undefined), 395-401. doi:10.12659/MSM.912341

[20] Chu Weiwei,Zhai Junyu,Xu Jieying et al. Continuous Light-Induced PCOS-Like Changes in Reproduction, Metabolism, and Gut Microbiota in Sprague-Dawley Rats.[J] .Front Microbiol, 2019, 10: 3145 .

[21] Barrea Luigi., Arnone Angela., Annunziata Giuseppe., Muscogiuri Giovanna., Laudisio Daniela., Salzano Ciro., Pugliese Gabriella., Colao Annamaria., Savastano Silvia.(2019). Adherence to the Mediterranean Diet, Dietary Patterns and Body Composition in Women with Polycystic Ovary Syndrome (PCOS). Nutrients, 11(10), undefined. doi:10.3390/nu11102278

[22] Lloyd-Price J, Mahurkar A, Rahnavard G, Crabtree J, Orvis J, Hall AB, Brady A, Creasy HH, McCracken C, Giglio MG, McDonald D, Franzosa EA, Knight R, White O, Huttenhower C. 2017. Strains, functions and dynamics in the expanded Human Microbiome Project. Nature 550:61-66.

https://doi.org/10.1038/nature23889.

[23] Walters Kirsty A., Gilchrist Robert B., Ledger William L., Teede Helena J., Handelsman David J., Campbell Rebecca E.(2018). New Perspectives on the Pathogenesis of PCOS: Neuroendocrine Origins. Trends Endocrinol. Metab., 29(12), 841-852. doi:10.1016/j.tem.2018.08.005

[24] Lim Siew S., Hutchison Samantha K., Van Ryswyk Emer., Norman Robert J., Teede Helena J., Moran Lisa J.(2019). Lifestyle changes in women with polycystic ovary syndrome. Cochrane Database Syst Rev, 3(undefined), CD007506. doi:10.1002/14651858.CD007506.pub4

[25] Zheng Yanhua., Yu Jingwei., Liang Chengjie., Li Shuna., Wen Xiaohui., Li Yanmei.(2020). Characterization on gut microbiome of PCOS rats and its further design by shifts in high-fat diet and dihydrotestosterone induction in PCOS rats. Bioprocess Biosyst Eng, undefined(undefined), undefined. doi:10.1007/s00449-020-02320-w

[26] Sanchez-Garrido Miguel A., Tena-Sempere Manuel.(2020). Metabolic dysfunction in polycystic ovary syndrome: Pathogenic role of androgen excess and potential therapeutic strategies. Mol Metab, 35(undefined), 100937. doi:10.1016/j.molmet.2020.01.001

[27] Porchia Leonardo M., Hernandez-Garcia Samantha Celeste., Gonzalez-Mejia M Elba., López-Bayghen Esther.(2020). Diets with lower carbohydrate concentrations improve insulin sensitivity in women with 
polycystic ovary syndrome: A meta-analysis. Eur. J. Obstet. Gynecol. Reprod. Biol., 248(undefined), 110117. doi:10.1016/j.ejogrb.2020.03.010

[28] Dumont, A., Robin, G., Catteau-Jonard, S., and Dewailly, D. (2015). Role of anti-müllerian hormone in pathophysiology, diagnosis and treatment of polycystic ovary syndrome: a review. Reprod. Biol. Endocrinol. 13:137. doi: 10.1186/s12958-015-0134-9

[29] Dewailly Didier., Robin Geoffroy., Peigne Maëliss., Decanter Christine., Pigny Pascal., Catteau-Jonard Sophie.(2016). Interactions between androgens, FSH, anti-Müllerian hormone and estradiol during folliculogenesis in the human normal and polycystic ovary. Hum. Reprod. Update, 22(6), 709-724. doi:10.1093/humupd/dmw027

[30] Kicinska Anna., Kampa Rafał P., Daniluk Jan., Sek Aleksandra., Jarmuszkiewicz Wieslawa., Szewczyk Adam., Bednarczyk Piotr.(2020). Regulation of the Mitochondrial BK Channel by the Citrus Flavonoid Naringenin as a Potential Means of Preventing Cell Damage. Molecules, 25(13), undefined. doi:10.3390/molecules25133010

[31] Insenser M, Murri M, Del Campo R, Martínez-García MÁ, Fernández-Durán E, Escobar-Morreale HF. 2018. Gut microbiota and the polycystic ovary syndrome: influence of sex, sex hormones, and obesity. J Clin Endocrinol Metab 103:2552-2562. https://doi.org/10.1210/jc.2017-02799.

[32] O'Callaghan A, van Sinderen D. 15 June 2016, posting date. Bifidobacteria and their role as members of the human gut microbiota. Front Microbiol https://doi.org/10.3389/fmicb.2016.00925.

[33] Qi Xinyu., Yun Chuyu., Sun Lulu., Xia Jialin., Wu Qing., Wang Ying., Wang Lina., Zhang Yangming., Liang Xianyi., Wang Liying., Gonzalez Frank J., Patterson Andrew D., Liu Huiying., Mu Liangshan., Zhou Zehong., Zhao Yue., Li Rong., Liu Ping., Zhong Chao., Pang Yanli., Jiang Changtao., Qiao Jie.(2019). Gut microbiota-bile acid-interleukin-22 axis orchestrates polycystic ovary syndrome. Nat. Med., 25(8), 12251233. doi:10.1038/s41591-019-0509-0

[34] Cai Wei., Xu Jixiong., Li Gang., Liu Tao., Guo Xiali., Wang Huajie., Luo Liping.(2020). Ethanol extract of propolis prevents high-fat diet-induced insulin resistance and obesity in association with modulation of gut microbiota in mice. Food Res. Int., 130(undefined), 108939. doi:10.1016/j.foodres.2019.108939

[35] Insenser María., Murri Mora., Del Campo Rosa., Martínez-García M Ángeles., Fernández-Durán Elena., Escobar-Morreale Héctor F.(2018). Gut Microbiota and the Polycystic Ovary Syndrome: Influence of Sex, Sex Hormones, and Obesity. J. Clin. Endocrinol. Metab., 103(7), 2552-2562. doi:10.1210/jc.2017-02799

[36] Li Jingjing., Ma Yunlong., Bao Zhiwei., Gui Xiaohua., Li Andria N., Yang Zhongli., Li Ming D.(2020). Clostridiales are predominant microbes that mediate psychiatric disorders. J Psychiatr Res, 130(undefined), 48-56. doi:10.1016/j.jpsychires.2020.07.018 
[37] Yang Hua., Xiao Yingping., Wang Junjun., Xiang Yun., Gong Yujie., Wen Xueting., Li Defa.(2018). Core gut microbiota in Jinhua pigs and its correlation with strain, farm and weaning age. J. Microbiol., 56(5), 346-355. doi:10.1007/s12275-018-7486-8

[38]cZhang Jun., Luo Dan., Lin Zhiming., Zhou Wenying., Rao Jialing., Li Yuanqing., Wu Jianhao., Peng Hui., Lou Tanqi.(2020). Dysbiosis of gut microbiota in adult idiopathic membranous nephropathy with nephrotic syndrome. Microb. Pathog., undefined(undefined), 104359. doi:10.1016/j.micpath.2020.104359

[39] Michaudel Chloé., Sokol Harry.(2020). The Gut Microbiota at the Service of Immunometabolism. Cell Metab., undefined(undefined), undefined. doi:10.1016/j.cmet.2020.09.004

[40] Jenab Anahita., Roghanian Rasoul., Emtiazi Giti.(2020). Bacterial Natural Compounds with AntiInflammatory and Immunomodulatory Properties (Mini Review). Drug Des Devel Ther, 14(undefined), 3787-3801. doi:10.2147/DDDT.S261283

[41] Hori Tetsuji., Matsuda Kazunori., Oishi Kenji.(2020). Probiotics: A Dietary Factor to Modulate the Gut Microbiome, Host Immune System, and Gut-Brain Interaction. , 8(9), undefined.

doi:10.3390/microorganisms8091401

[42] Wang Mingfei., Li Leping., Chen Yuezhi., Lian Guodong., Wang Jinshen., Zhang Jizhun., Shan Keshu., Shang Liang., Tian Feng., Jing Changqing.(2020). Role of Gut Microbiome and Microbial Metabolites in Alleviating Insulin Resistance After Bariatric Surgery. Obes Surg, undefined(undefined), undefined. doi:10.1007/s11695-020-04974-7

[43] Canfora EE, Jocken JW, Blaak EE. 2015. Short-chain fatty acids in control of body weight and insulin sensitivity. Nat Rev Endocrinol 11:577-591. https://doi.org/10.1038/nrendo.2015.128.

\section{Figures}



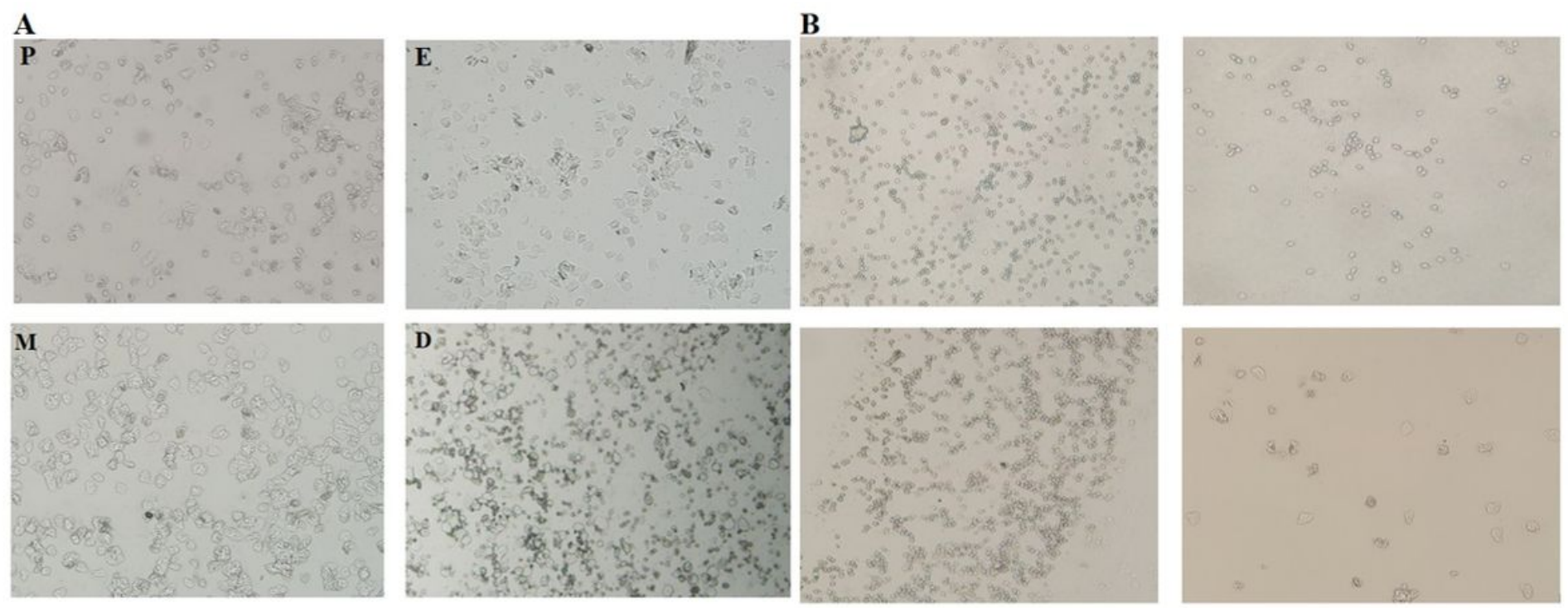

Figure 1

Test results of vaginal exfoliated cells.(A) vaginal exfoliated cells in normal control rats (regular estrus cycle), including proestrus period (mainly with nuclear epithelial cells); estrus period (mainly with nonnuclear keratinocytes); metestrus (nucleated epithelial cells, non-nucleated keratinocytes and leukocytes coexist); diestrus (mainly neutrophils).(B) disorder of the estrus cycle, the smear of vaginal exfoliated cells in letrozole-induced PCOS rats modeling (persistent Diestrus interval). P: Proestrus ; E: Estrus; M: Metestrus ;D: Diestrus.

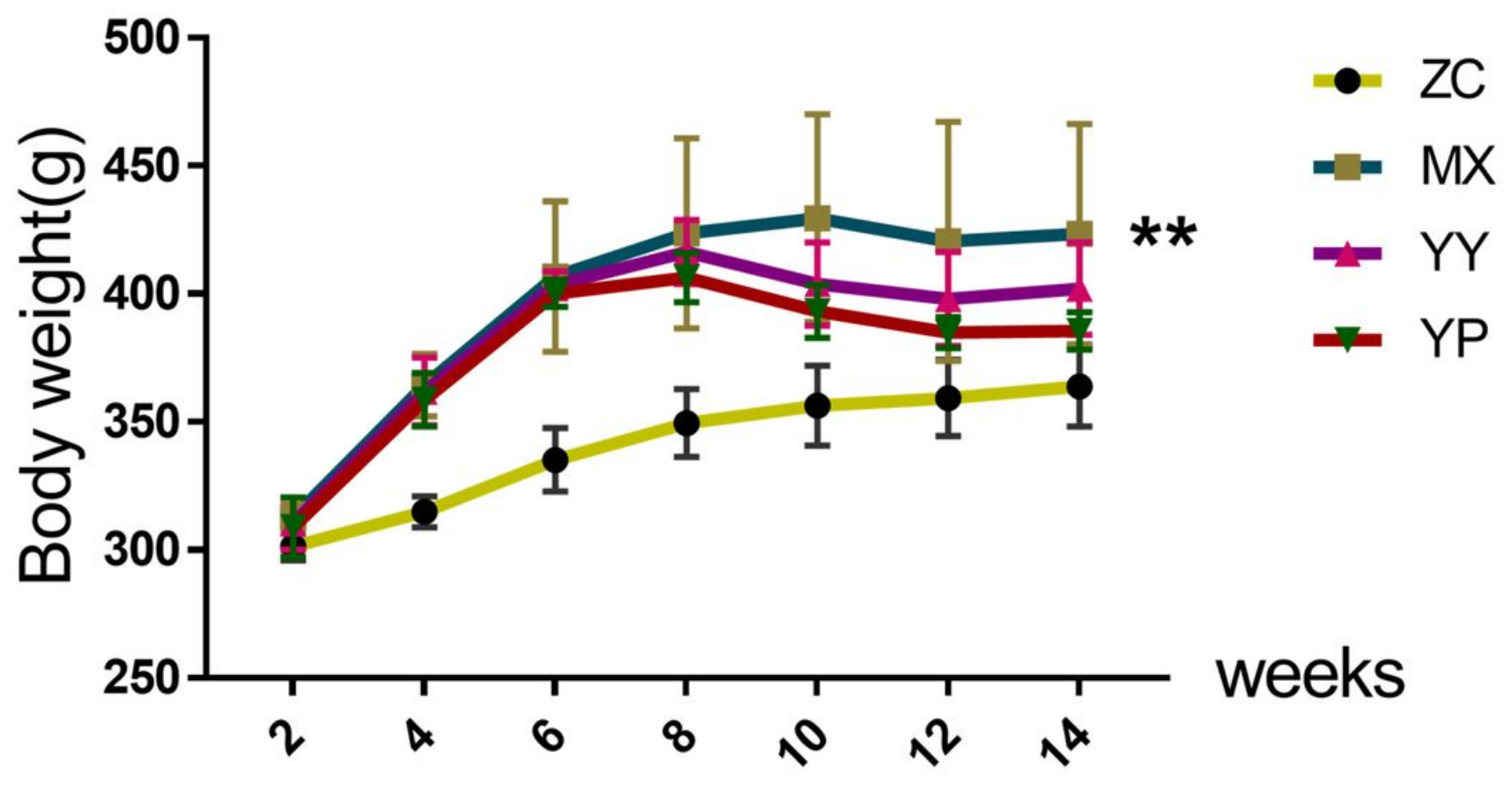




\section{Figure 2}

Line chart of the effect of naringenin on letrozole-induced PCOS in rats, the abscissa represents the intervention time (weeks)( the first 2 weeks is the adaptation period, 2-6 weeks is the letrozole-induced PCOS period, and 6-14 weeks is the naringenin treatment period), and the ordinate represents the weight, which are distinguished by different colors, as shown in the figure. ZC: normal group, MX: PCOS group, YY: Diane-35 group, YP: naringenin group.

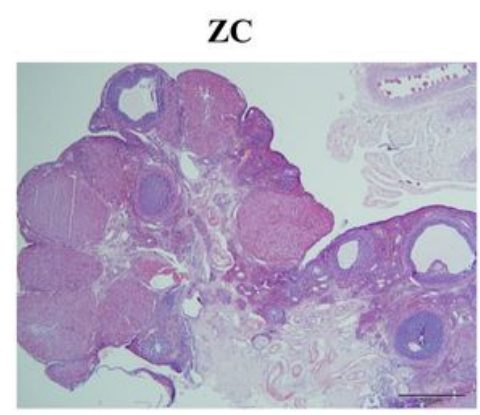

ZC

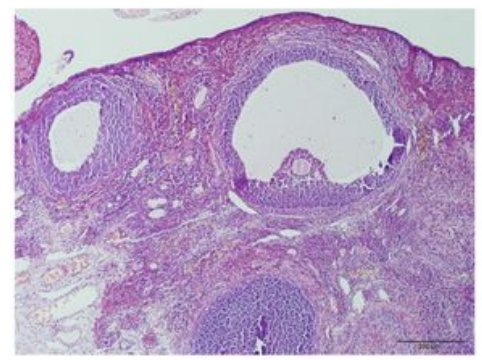

ZC

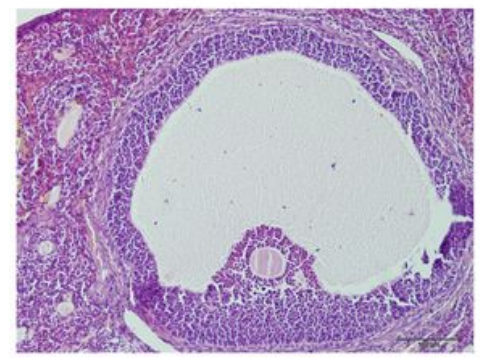

MX

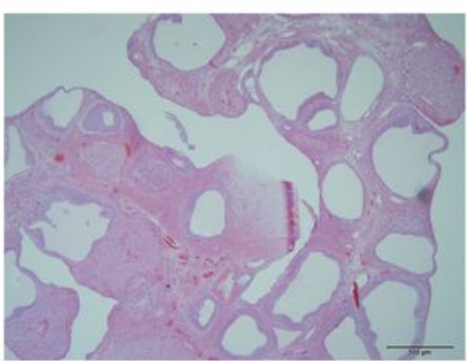

MX

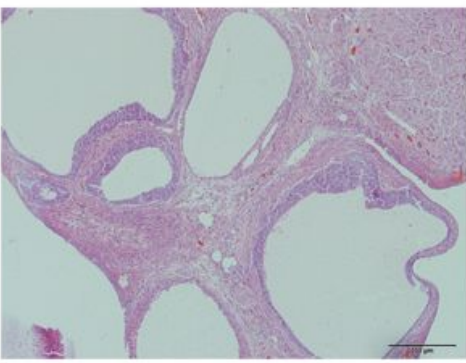

MX

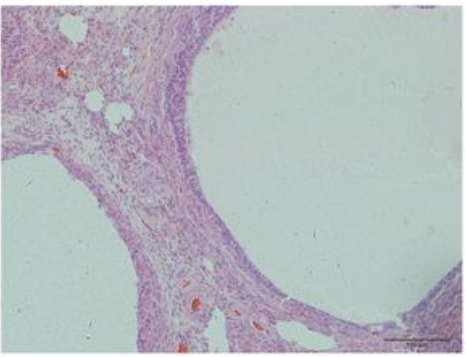

YY

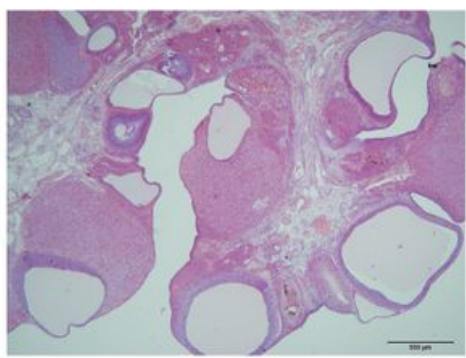

YY

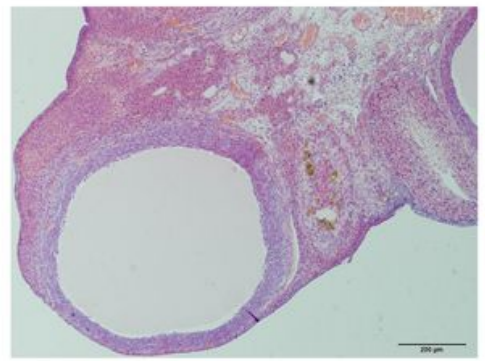

YY

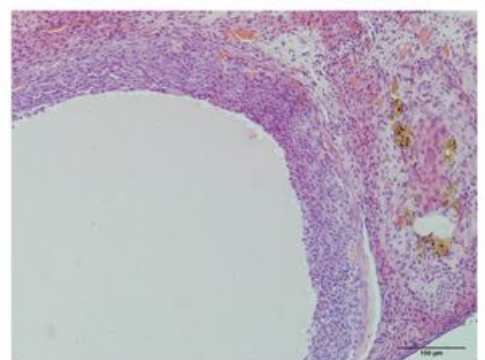

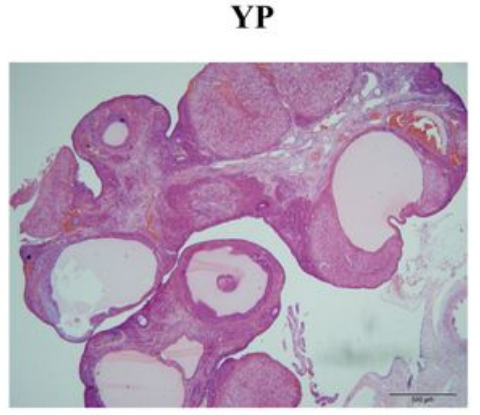

YP

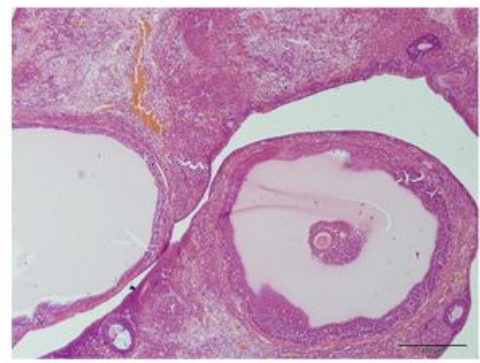

YP

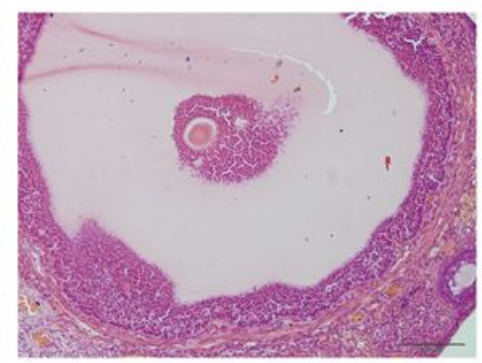

\section{Figure 3}

Section of the overy from each experimental group (H\&E, scale bar=500um,200 um,100 um). ZC: normal group, MX: PCOS group, YY: Diane-35 group, YP: naringenin group. 

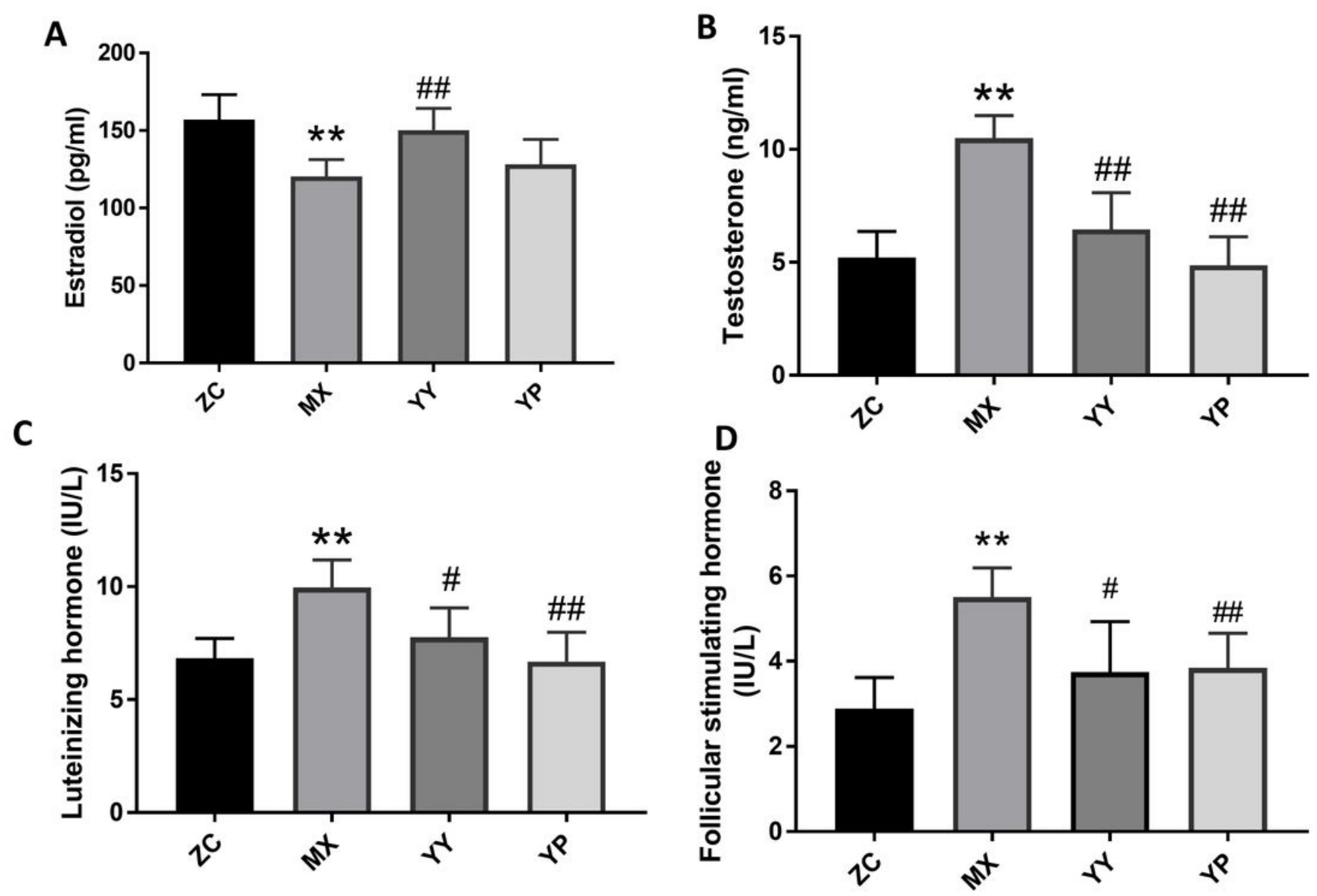

Figure 4

Effect of treatment with quinoa on serum hormone levels in letrozole-induced PCOS rats. Serum hormonal levels were using a radio immunosorbent assay such as (A) E2,(B) T,(C)LH,(D)FSH. All values represent means \pm SEM; $n=6$ per group; ${ }^{*} p<0.05$, ${ }^{*} p<0.01 v$ s control; $\# p<0.05, \# \# p<0.01 v s$ PCOS. ZC: normal group; MX: PCOS group; YY: Diane-35 group; YP: naringenin group. 

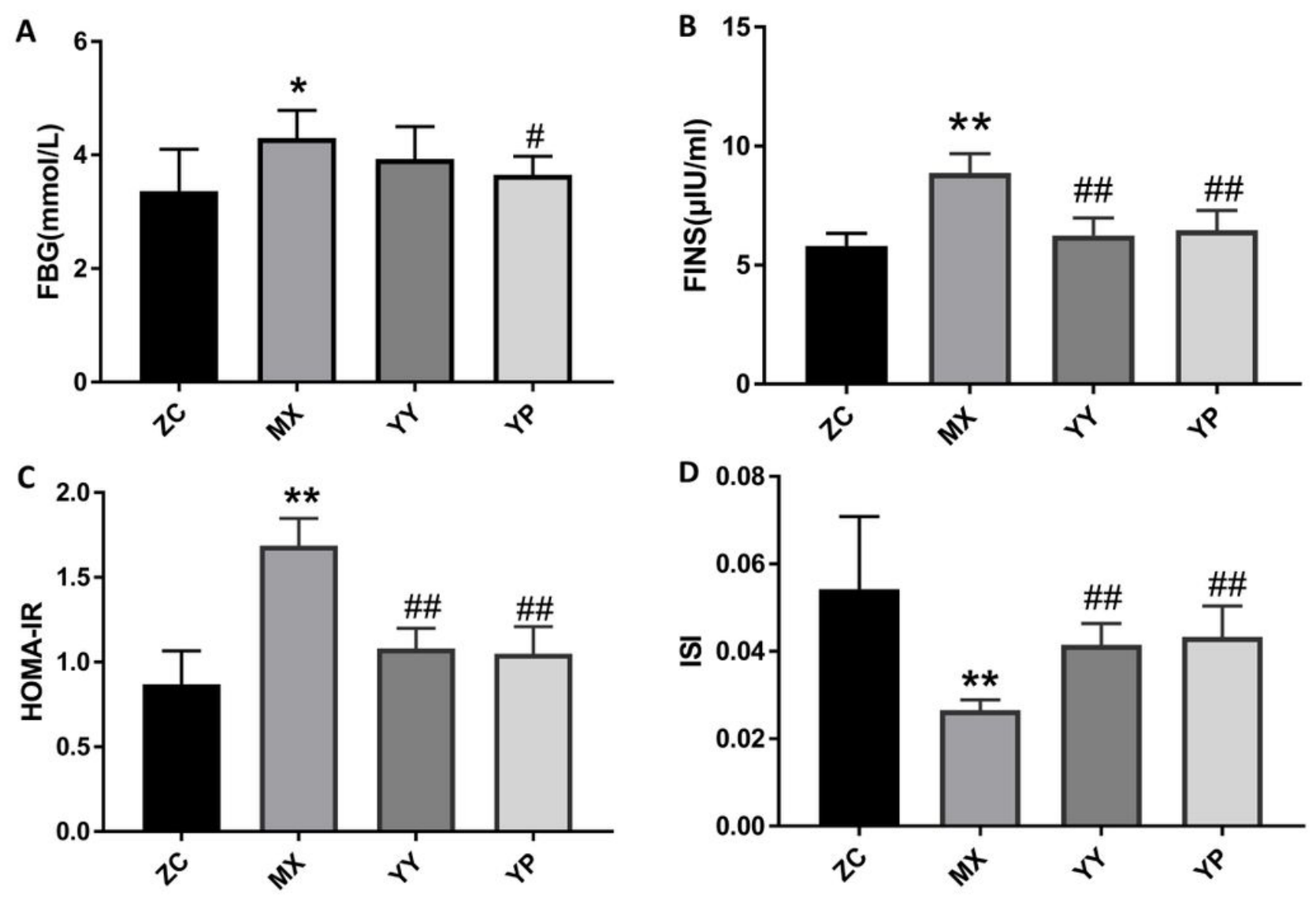

Figure 5

Effect of quinoa on glucose metabolism in letrozole-induced PCOS rats. Serum hormonal levels such as (A) FBG was measured by special blood glucose meter. (B) FINS was measured using a radio immunosorbent assay. (C)HOMA-IR and (D)ISI were calculated. All values represent means $\pm S E M ; n=6$ per group; ${ }^{*}<<0.05,{ }^{* \star} p<0.01$ vs control; $\# p<0.05, \# \# p<0.01$ vs PCOS. ZC: normal group, MX: PCOS group, YY: Diane-35 group, YP: naringenin group. 

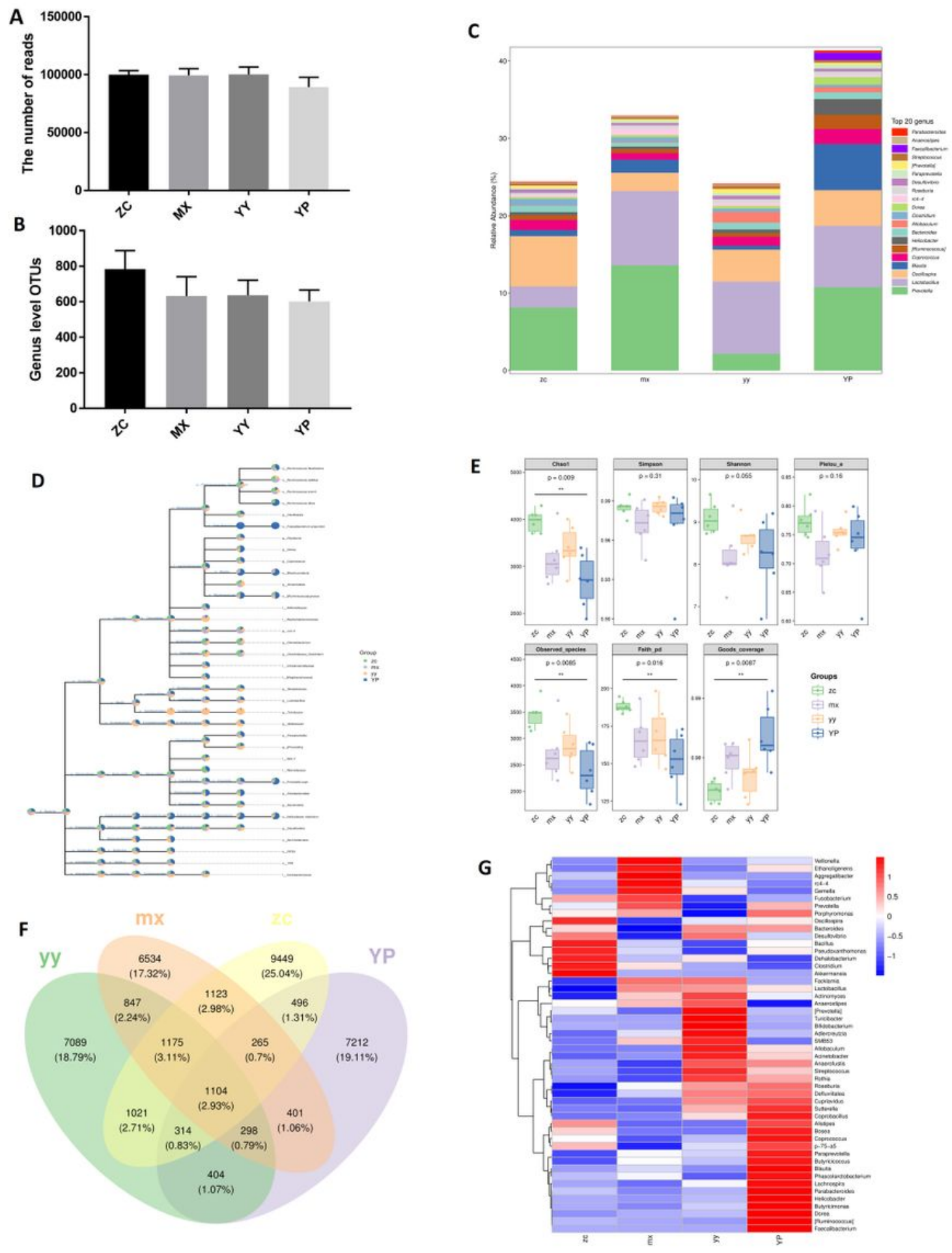

\section{Figure 6}

The intestinal microbiota exhibited an inconsistent response to naringenin treatment on gut microbiome of PCOS of letrozole-induced rats model. (A)usable raw reads of the intestinal microbiota. (B) OTU-level rarefaction (OTUs). (C) Taxonomic compositions of bacterial communities in the genus level in four groups. (D) taxonomic_tree (threshold value $0.5 \%$ ) shows the composition ratio of the taxon in each group. The larger the fan area, the abundance of the taxon in the corresponding group higher. (E)The 
alpha diversity, the different of the color represents the difference group, values are shown by box-plot. Box represents the interquartile range. The line inside the box represents the median. And whiskers denote the minimum and maximum value. $* *$ Adjusted $\mathrm{P}<0.01$ (Kruskal-Wallis test). $(\mathrm{F})$ Venn diagram, each ellipse represents a group, the overlapping area between the ellipses indicates the total ASV/OTU between the groups, and the number of each block indicates the number of ASV/OTU contained in the block. (G) A heat map representing the intestinal microbial changes at the genus level in four groups, the degrees of depth of the color represents the relative abundances of the related genus (in the figure, the red color block indicates that the genus is more abundant in the sample than other samples, and the blue color block is the opposite). $n=6$ per group. ZC: normal group, MX: PCOS group, YY: Diane-35 group, YP: naringenin group. 